\title{
Journal of Pregnancy and Child Health
}

\section{Using Intervention Mapping for a Needs Assessment on Preconception Care in Suriname: The Perisur Project}

Marije E Kamphuis ${ }^{1}$, Dineke G Korfker ${ }^{2}$, Symone B Detmar ${ }^{2}$, Manodj P Hindori ${ }^{3,4}$, Magda M Boere-Boonekamp ${ }^{1}$, Hindrik Vondeling ${ }^{1,4}$ and Ashna D HindoriMohangoo $2,4,6^{*}$

${ }^{1}$ Department Health Technology and Services Research, Institute of Innovation and Governance Studies, University of Twente, Enschede, The Netherlands

${ }^{2}$ Netherlands Organization for Applied Scientific Research, TNO Healthy Living, Department Child Health, Leiden, The Netherlands

${ }^{3}$ Sint Vincentius Hospital, Paramaribo, Suriname

${ }^{4}$ Perisur Foundation, Paramaribo, Suriname

${ }^{5}$ Centre of Health Economics Research, University of Southern Denmark, Odense, Denmark

${ }^{6}$ Anton de Kom University of Suriname, Faculty of Medical Sciences, Department Public Health, Paramaribo, Suriname

*Corresponding author: Ashna D Hindori Mohangoo, Netherlands Organization for Applied Scientific Research, TNO Healthy Living, Department Maternal and Child Health, Leiden, The Netherlands, Tel: 31611783003; Fax 31 888660613; E-mail: ashna.mohangoo@tno.nl

Received date: June 25, 2016; Accepted date: June 27, 2016; Published date: June 30, 2016

Copyright: (c) 2016 Kamphuis ME, et al. This is an open-access article distributed under the terms of the Creative Commons Attribution License, which permits unrestricted use, distribution, and reproduction in any medium, provided the original author and source are credited.

\begin{abstract}
Background: Every year approximately 10,000 babies are born in Suriname of which an estimated 400 die in the perinatal period. The main purpose of the Perisur project is to improve perinatal outcomes and improve under-five and maternal health. This study focused on introducing preconception care in Suriname.
\end{abstract}

Methods: Intervention Mapping was used as guiding framework and we focused on its first the needs assessment.

Step: A mixed method study with a sequential design was performed. The PRECEDE model served as tool to conceptualize the needs.

Results: Twenty-nine stakeholders responded to a web-based survey (response rate 41\%). Important preconception risk factors included hypertension, diabetes mellitus and teenage pregnancies. Six interviews were conducted, identifying various perspectives on preconception problems and its causes. Lack of pregnancy planning was assessed as most alarming risk behaviour, causing two preconception problems: unplanned and teenage pregnancies. A sample of thirty-nine potential end-users (pregnant women) completed a survey on preconception counselling: $76 \%$ found preconception counselling important and $46 \%$ considered going. As a final step, we derived the following program goals: improve education, (equal) access to care services, risk awareness and risk perception; and increase the number of women preparing their pregnancy (e.g. via preconception counselling).

Conclusion: This study made a first step in developing a tailored preconception care program to improve perinatal outcomes in Suriname. The methodological approach in this study provides a basis for future preconception care interventions within the Perisur project.

Keywords: Millennium development goals; Preconception care; Intervention mapping; Needs assessment; Perisur

\section{Introduction}

Every year approximately 10,000 babies are born in Suriname, of which an estimated 400 die in the perinatal period (perinatal mortality rate of 40 per 1000 births). This rate is based on preliminary results of a perinatal and infant mortality survey (A.D. Hindori-Mohangoo, personal communication, November 11, 2014). The World Health Organization (WHO) [1] reported that the perinatal mortality rate of Suriname is relatively high compared to other South American countries. Perinatal mortality is largely the result of prematurity, low birth weight, severe birth defects and a low Apgar score at birth. In addition to these factors, transverse or breech presentation at delivery, high maternal age (35+ years), teenage pregnancies and multiple pregnancies contribute to the perinatal mortality rate in Suriname (A.D. Hindori-Mohangoo, personal communication, November 11, 2014). Cousens et al. state that Suriname will not achieve the Millennium Development Goal 4 (MDG 4) of reducing under-five mortality by two-thirds in a 15 year period ending this year 2015 . The majority of under-five mortality (87\%) in Suriname occurs in the first year of life, and of this mortality, $64 \%$ occurs in the early neonatal period (United Nations Development Program 2014). Therefore, interventions to improve perinatal outcomes are important to achieve MDG 4.

In 2012, the Sint Vincentius Hospital in Suriname and the Netherlands Organization for Applied Scientific Research organized an expert meeting to discuss the national issues regarding the high perinatal mortality. This resulted in the establishment of a new perinatal-intervention-based project, Perisur. Selected interventions include the introduction of internal perinatal audits, group prenatal 
Citation: Kamphuis ME, Korfker DG, Detmar SB, Hindori MP, Boere-Boonekamp MM, et al. (2016) Using Intervention Mapping for a Needs Assessment on Preconception Care in Suriname: The Perisur Project. J Preg Child Health 3: 263. doi:10.4172/2376-127X.1000263

Page 2 of 6

care and preconception care. The latter is the focus of this study. Preconception care includes "interventions that aim to identify and modify biomedical, behavioural and social risks to a woman's health or pregnancy outcome through prevention and management" [1]. Worldwide awareness on preconception care is growing [2-5]; however preconception care is still a weak link in the global continuum of care, since care usually starts in the antenatal period [6-8]. However, the most critical time of embryonic development is before a woman knows she is pregnant [9]. The strength of evidence for preconception care varies widely and its quantity is low. Preconception care is currently not embedded in the general service provision of (public) health care facilities in Suriname.

We selected the Intervention Mapping framework of Bartholomew et al. [10] as guiding tool for program development. In Intervention Mapping basic tools are systematically applied in six fundamental steps of program development (Figure 1). This paper elaborates on the first step, encompassing the planning and performance of a needs assessment on preconception care.

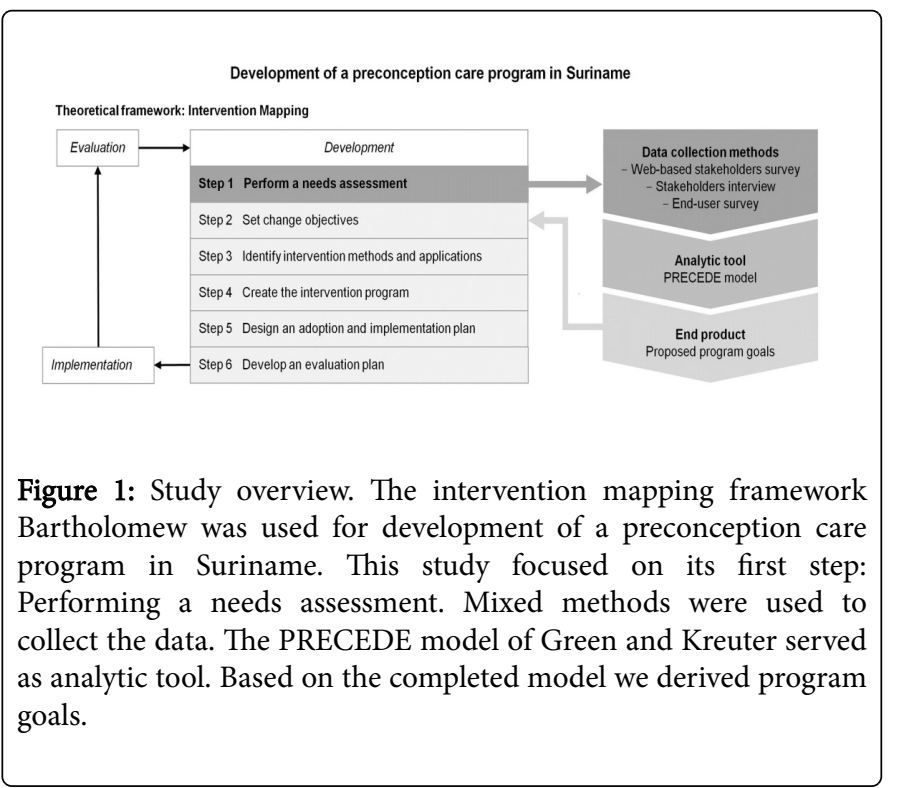

\section{Methods}

\section{Design}

During summer 2013 a mixed methods study with an explanatory sequential design was conducted. Figure 1 presents the choice and sequence of the methods used. All tasks described in Intervention Mapping step 1, the needs assessment, were executed on site. A stakeholder's survey was conducted up front to get a first impression of the preconception needs in Suriname. The needs assessment was completed with a stakeholder's interview, to improve our understanding of the preconception health problems and their underlying causes. We also surveyed potential end-users of preconception care to explore their potential commitment towards preconception care. This study was approved by the Medical Ethical Committee of the Ministry of Health in Suriname, reference number VG 020-2013, and has been performed in accordance with the ethical standards laid down in the 1964 Declaration of Helsinki and its later amendments. All participants gave their informed consent prior to their inclusion in the study [11].

The PRECEDE part of the PRECEDE-PROCEED model of Green and Kreuter [12] was used as analytic tool to conceptualize the needs assessed by the methods described above. As a final stage of step 1, the project team linked the needs, for which documentation was obtained, within the model to potential program goals. For example when "lack of risk awareness" was assessed as a need, this was translated to the following program goal: "increasing risk awareness".

\section{Data collection}

\section{Web-based stakeholders survey}

A web-based survey was sent to 70 local stakeholders that were part of the project team's network (e.g. first and second line care providers and managers, and relevant governmental employees). Professionals were asked to state their background and to prioritize to the presented preconception risk factors. The preconception risk factors were based on a list of the WHO [13] supplemented with risk factors expected to be relevant to the Surinamese setting (Table 1).

\begin{tabular}{|c|c|}
\hline Risk areas & Risk factors \\
\hline 1. Obstetric history & (Induced) abortions, perinatal mortality, congenital anomalies, maternal complications \\
\hline 2. Planning of pregnancy & Teenage pregnancy, short interval between pregnancies, pregnancy at older age $(35+)$, unplanned pregnancy \\
\hline 3. Fertility problems & Reduced fertility or infertility, limited or no treatment options \\
\hline 4. Genetic or familial disorders & Chromosomal defects, sickle cell disease, thalassemia \\
\hline 5. Chronic diseases & Hypertension, diabetes mellitus type II, epilepsy, depression/psychosis \\
\hline 6. Medication use & Anti-diabetic, antiepileptic \\
\hline 7. Sexually transmitted diseases & Chlamydia, gonorrhoea, syphilis, HIV \\
\hline 8. Vaccine-preventable infections & Rubella, tetanus, hepatitis B \\
\hline 9. Nutritional condition & Folic acid insufficiency, iron deficiency anaemia, maternal overweight, maternal underweight \\
\hline 10. Environmental factors & Poor residential living conditions, professional risks, pesticides, lead and mercury pollution \\
\hline
\end{tabular}


Citation: Kamphuis ME, Korfker DG, Detmar SB, Hindori MP, Boere-Boonekamp MM, et al. (2016) Using Intervention Mapping for a Needs Assessment on Preconception Care in Suriname: The Perisur Project. J Preg Child Health 3: 263. doi:10.4172/2376-127X.1000263

Page 3 of 6

\begin{tabular}{|l|l|}
\hline 11. Lifestyle factors & Tobacco use, alcohol use, drug use, physical inactivity \\
\hline 12. Domestic violence & Domestic violence \\
\hline 13. Female genital mutilation & Female genital mutilation \\
\hline
\end{tabular}

Table 1: Preconception risk areas as defined by the World Health Organization (WHO) (2013). The risk factor examples presented by the WHO were supplemented for the Surinamese context and used as input for the web-based stakeholders survey.

These risk factors were scored on a five-point Likert-scale from very low priority ( 1 point) to very high priority ( 5 points). Survey Monkey was used to collect the data online. An overview of the survey content is presented in the Supplement.

\section{Stakeholders interview}

We planned to interview representatives of the same stakeholder groups. We preferred to interview at least two representatives of each group in order to reach saturation. The interview candidates were recruited via email, telephone or via personal contact. The questions of the structured interviews were based on the topics presented in the PRECEDE model.

\section{Written survey among potential end-users}

Finally the needs of the future target group were explored. The survey used was derived from an existing Dutch survey [12] and tailored to the Surinamese context. Using seven questions women were asked about their planned behaviour towards preconception counselling, whereby the attitude - social influence - self efficacy model (ASE model) of de Vries [13] was applied. The survey was conducted among pregnant women (potential target group in a subsequent pregnancy). The women were easily accessible since they visited the cooperating hospital for a routine antenatal check. During a week in July 2013, all women in the waiting room were asked to fill in the survey. In case of illiteracy, the questions were read out by an officiating nurse. Women who could barely/not speak the Dutch, English or Sranan Tongo (local) language were not included.

\section{Data analysis}

Average scores were calculated for each of the preconception risk factors. Differences in mean scores between first and second line health care workers and between private and public health care workers were tested using one-way ANOVA. The significance level was set at $\mathrm{p}<0.05$. Statistical analyses were performed using the statistical package for social sciences (SPSS Inc, Chicago, IL, USA), version 20.0 for Windows. The stakeholder interview results were coded, listed per question and analysed.

\section{Results}

\section{Web-based stakeholders survey and stakeholders interviews}

Twenty-nine out of 70 stakeholders (41\%) responded to the webbased survey, including general practitioners (GPs), governmental employees, hospital managers, nurses, obstetricians/gynaecologists, and paediatricians. Twenty-one per cent of respondents were primary health care workers, $69 \%$ were hospital workers, and $10 \%$ had other backgrounds. From the hospital workers, $65 \%$ were employed in private hospitals and $35 \%$ in public hospitals. Figure 2 shows the average scores of the ranked preconception risk factors.

The overall mean score for all risk factors was $3.38 \pm 0.90$ out of 5 . Highest priority was assigned to pre-existent hypertension (4.31 \pm $0.43)$; diabetes mellitus type II ( $4.24 \pm 0.47)$, and teenage pregnancy $(4.17 \pm 0.46)$. No significant inter-group differences were observed between primary and hospital health care workers. Public hospital workers gave significantly higher priority to teenage pregnancies compared to private hospital workers ( 4.57 vs. $3.85 ; \mathrm{p}=0.027$ ).

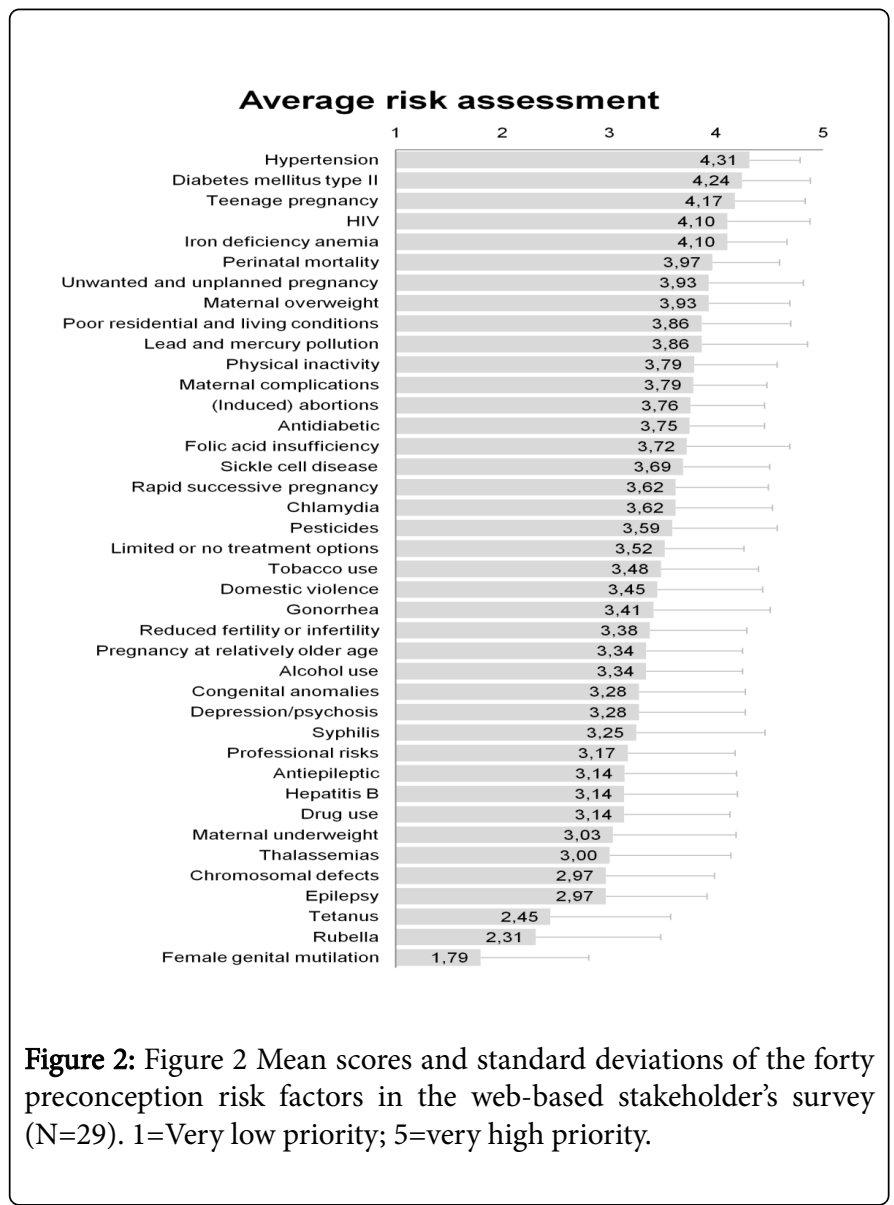

Six face-to-face interviews were conducted. The interviewees' backgrounds comprised three hospital managers, two medical specialists, and one government employee. Four of the interviewees worked for a private hospital, one for a public hospital and one for the government. The lack of pregnancy planning was considered the most alarming risk behaviour of Surinamese women. One interviewee stated: "When a woman starts planning her pregnancy, she is able to take pre-emptive measures to improve her pregnancy outcome if 
Citation: Kamphuis ME, Korfker DG, Detmar SB, Hindori MP, Boere-Boonekamp MM, et al. (2016) Using Intervention Mapping for a Needs Assessment on Preconception Care in Suriname: The Perisur Project. J Preg Child Health 3: 263. doi:10.4172/2376-127X.1000263

Page 4 of 6

needed". This input was used to enter the analytic model. The completed model (Figure 3 ) is explained on the basis of the following categories: (1) preconception problems, health outcomes, and populations at risk, (2) behaviour of risk groups and personal determinants, and (3) environmental factors.

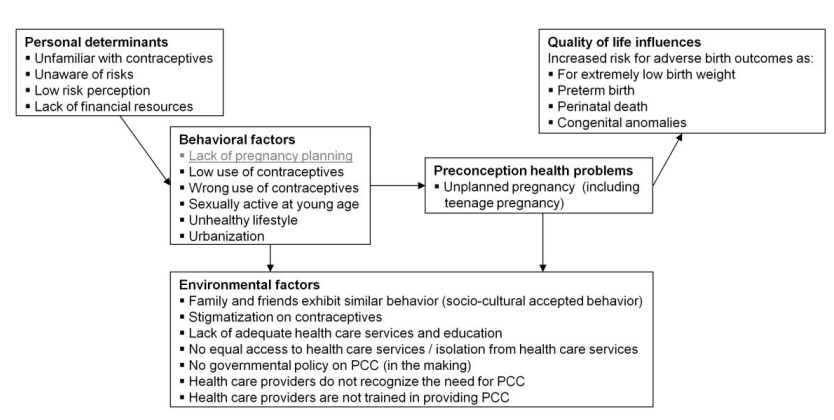

Figure 3: Mean scores and standard deviations of the forty preconception risk factors in the web-based stakeholder's survey $(\mathrm{N}=29) .1=$ Very low priority; $5=$ Very high priority.

\section{Preconception problems, health outcomes, and populations at risk}

According to one interviewee, pregnancy planning is only carried out by a minority of Surinamese women, which generally include women with a higher socio-economic status. Especially women with chronic diseases and teenage girls were mentioned to have increased pregnancy risks due to their non-planning behaviour. One interviewee gave the following example: "Women with diabetes should reset their insulin pump before or in the beginning of their pregnancy to optimize sugar balance for both mother and baby, however they are generally unaware of this". Two interviewees mentioned they see in their daily practice an increasing number of women with chronic diseases. Obesity and lack of physical exercise were mentioned as significant contributors to this.

\section{Behaviour of risk groups and personal determinants}

During the interviews, low and wrong use of contraceptives, sexual activity at young age, and an unhealthy lifestyle were mentioned as risk behaviours next to the lack of pregnancy planning. Two interviewees also emphasized the pattern of urbanization in recent years. Personal determinants underlying these risk behaviours of Surinamese women (including teenage girls) were considered the unfamiliarity with contraceptives, but moreover the unawareness of women of their preconception risks and their low risk perception. One interviewee mentioned that teenage girls lack understanding of the need to prevent pregnancy. Also lack of financial resources was mentioned.

\section{Environmental factors}

One of the environmental factors contributing to preconception problems included the lack of support from family and friends for the need to prevent teenage pregnancy, since teenage pregnancy is a socioculturally accepted phenomenon among certain communities. Other environmental factors mentioned included the stigmatization of contraceptives and the lack of sexual and reproductive health education in schools (taboo subject). Poverty in terms of education and living standards is relatively impaired and unequally geographically distributed. Due to the remoteness of the interior and coastal areas, basic and specialized health care services are not for everyone within reach (e.g. selling points for contraceptives and family planning consultation). The absence of national guidelines on adequate pregnancy care, including preconception care, was mentioned by four interviewees. One of them said the majority of health care professionals are not trained in providing adequate preconception care. The following was stated: "Women with a child wish in combination with an obstetric history of perinatal death are seldom referred to a specialist via their GP, since most GPs do not recognize the need for it".

\section{Written survey among potential end-users}

Thirty-nine out of 41 pregnant women completed the questionnaire. Five women were excluded because of the language barrier. Although $67 \%$ of the respondents had never heard of preconception counselling, the majority stated they would consider going before pregnancy (certainly: $46 \%$ and probably: $10 \%$ ). $76 \%$ of respondents replied they found preconception counselling (very) important, and no one found it unimportant.

\section{Linkage to program goals}

Based on the results we arrived at the proposed program goals presented in Figure 4. We made a distinction between the societal and community/organizational intervention level. 


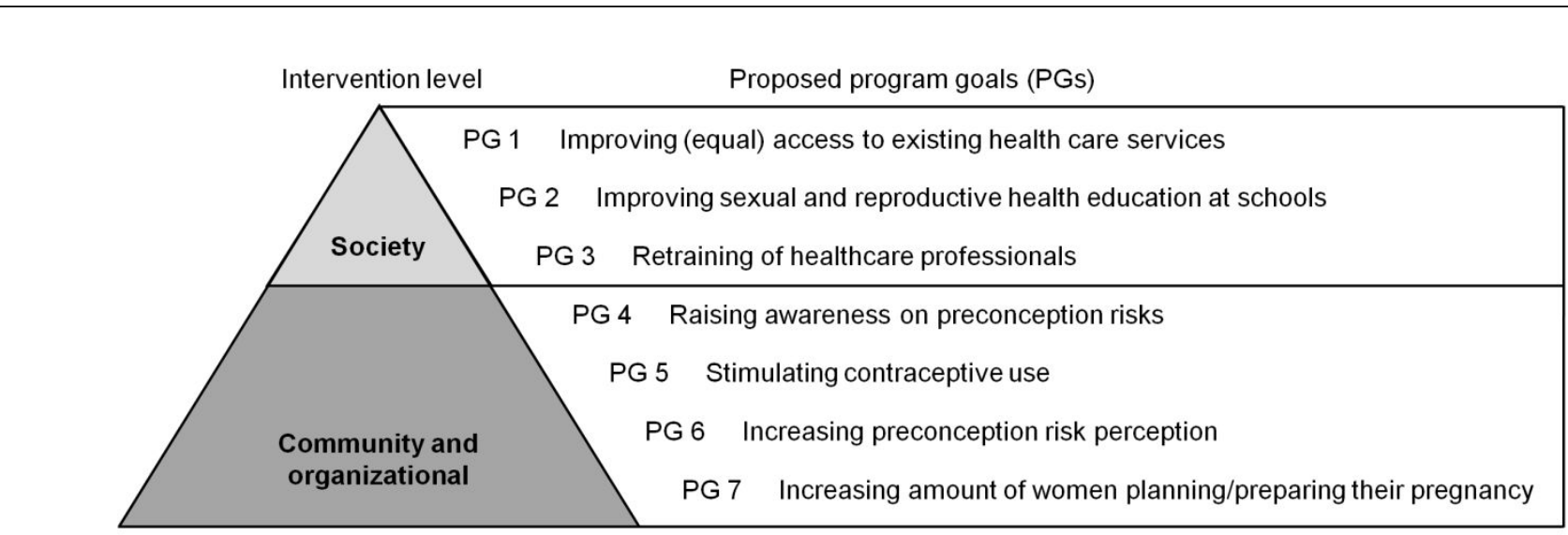

Figure 4: Proposed program goals directly derived from the completed analytic model.

\section{Discussion}

With this study, a first step in developing a preconception care program in Suriname was made by performing a needs assessment with surveys and interviews among local stakeholders and potential end-users. Unplanned and teenage pregnancies were assessed as the main preconception health problems, causing a (preventable) increased risk of adverse birth outcomes. Populations at risk were considered all women of childbearing age, especially teenage girls and women with chronic disease. Resulting program goals included: improving education and (equal) access to health care services, increasing risk awareness and risk perception, and increasing the number of women preparing their pregnancy.

The findings from our needs assessment are in line with a recently published United Nations Development Progress Report on Suriname [14]. Next steps towards achieving the MDG 4, as identified in the report, included: the implementation of sexual and reproductive health policy and the implementation of a national safe motherhood and new-born health action plan. With our preconception care program we intend to address several challenges appointed in the report in cooperation with the local government.

A strength of this study is the use of a structured approach for program development and decision-making, like Intervention Mapping, to make a first assessment of preconception needs in Suriname. The approach has the additional advantage of reproducibility, for instance, our structured and transparent way of designing preconception care can serve as an example for other lowand middle-income countries. We believe our program is useful because according to a meeting report of the WHO [15] there is a worldwide need for implementation strategies and increasing coverage of (existing) cost-effective preconception care interventions. Besides, one of the needs that emerged from the report is to scale the quantity of evidence for preconception care.

Since local stakeholders from various backgrounds prioritized the preconception risk factors by completing the web-based survey, the results of this part of our study gave valuable information on the preconception needs in Suriname. Unfortunately, due to time and budget restrictions not all stakeholder groups could eventually be interviewed. The completed PRECEDE model was largely based on outcomes of the six interviews and as four of these were affiliated with private hospital workers, this limits the generalizability of our results. In addition the selection procedures for the stakeholders survey and interviews can cause selection bias. In future studies the needs assessment should be extended and e.g. include more primary care health professionals, policy makers and representatives of relevant non-governmental organizations.

Preconception counselling is considered a potential delivery method for preconception care. We noticed from the survey among potential end-users that most women responded positively towards preconception counselling. Since it was the first time these women have ever participated in scientific research, it is difficult to say how reliable the answers were. Thereby, some questions were found difficult to answer (left blank, or were questioned). In order to get a better indication of their planned behaviour, future research should reconsider how to assess their planned behaviour in the best way. The end-user survey restricted us to women/couples living nearby the coordinating private hospital. Nevertheless, this might be an easily accessible target group to start with for the preconception care program. According to Rogers [15] women with a relatively higher socio-economic status often become early adapters of an intervention program. In the following steps of Intervention Mapping we might consider to first focus on these women, to create acceptance and then scale-up after successful implementation [16-18].

\section{Conclusion}

Within the Perisur project a first step has been made in the development of a preconception care program in Suriname. The program was tailored to Surinamese needs to improve perinatal outcomes and thus to contribute to realizing MDG4. The assessment resulted in a diverse, though limited, impression of the Surinamese needs. Nevertheless the study lays the foundation for the next steps in implementing a full-scale preconception program in Suriname, which focuses on (i) improving education and (equal) access to care services, (ii) increasing awareness and perception on preconception risks, and (iii) increasing the number of women/couples who plan their pregnancy and take pre-emptive measures in order to prepare for a healthy pregnancy. The methodological approach in this study provides a basis for future preconception care interventions within the Perisur project. 
Citation: Kamphuis ME, Korfker DG, Detmar SB, Hindori MP, Boere-Boonekamp MM, et al. (2016) Using Intervention Mapping for a Needs Assessment on Preconception Care in Suriname: The Perisur Project. J Preg Child Health 3: 263. doi:10.4172/2376-127X.1000263

Page 6 of 6

\section{Acknowledgement}

This study was carried out in the context of the MPH program of the Health Sciences Program, at the University of Twente, Enschede, The Netherlands. The authors thank the Sint Vincentius Hospital in Suriname and the Netherlands Organization for Applied Scientific Research (TNO) for supporting this study. These organizations initiated the Perisur project which is funded by TNO and the Twinning Facility Suriname Netherlands. We also thank the Ministry of Health in Suriname for giving us permission to conduct this study and we thank the Preparing for Life Initiative for their support. We are extremely grateful to all the women that participated in this study and all the local stakeholders who shared their knowledge. The authors declare that they have no conflict of interest.

\section{References}

1. Johnson K, Posner SF, Biermann J, Cordero JF, Atrash HK, et al. (2006) Recommendations to improve preconception health and health careUnited States. Report. National Center for Chronic Disease Prevention and Health Promotion.

2. Christiansen C, Mouli VC, Ogbaselassie L, Willumsen J, Mason E (2013) Meeting to develop a global consensus on preconception care to reduce maternal and childhood mortality and morbidity. Meeting report. World Health Organization.

3. Dean SV, Imam AM, Lassi ZS, Bhutta ZA (2011) A systematic review of preconception risks and interventions Report. Aga Khan University.

4. Health Council of the Netherlands (2007) Preconception care: A good beginning. Advisory report. Health Council of the Netherlands.

5. Jack BW, Atrash H, Coonrod DV, Moos MK, O’Donnell J, et al. (2008) The clinical content of preconception care: An overview and preparation of this supplement. Am J Obstet Gynecol 199: 266-279.

6. World Health Organization (2006) Neonatal and perinatal mortality: Country, regional and global estimates. Report. World Health Organization.
7. World Health Organization (2010) Packages of interventions for family planning, safe abortion care, maternal, new-born and child health. Report. World Health Organization.

8. Cousens S, Blencowe H, Stanton C, Chou D, Ahmed S, et al. (2011) National, regional, and worldwide estimates of stillbirth rates in 2009 with trends since 1995: A systematic analysis. Lancet 377: 1319-1330.

9. Atrash HK, Johnson K, Adams M, Cordero JF, Howse J (2006) Preconception care for improving perinatal outcomes: The time to act. Matern Child Health J p: 11.

10. Bartholomew LK, Guy SP, Kok G, Gottlieb NH, Fernández ME (2011) Planning health promotion programs - an intervention mapping approach. 3rd ed. San Fransisco: Jossey-Bass.

11. de Vries H, Dijkstra M, Kuhlman P (1988) Self-efficacy: The third factor besides attitude and subjective norm as predictor of behavioural intentions. Health Educ Res 3: 273-282.

12. Green LW, Kreuter MW (2005) Health program planning: an educational and ecological approach. 4th ed. New York: Mc-Graw Hill.

13. de Vries H, Dijkstra M, Kuhlman P (1988) Self-efficacy: The third factor besides attitude and subjective norm as predictor of behavioural intentions. Health Educ Res 3: 273-282.

14. UNDP, Surinamese Ministry of Foreign Affairs, General Bureau of Statistics. (2014) Government of the republic of Suriname, MDG progress report 2014.

15. Rogers EM (2003) Diffusion of innovations. 5th ed New York, London, Toronto, Sydney: Free Press.

16. The World Health Organization (2012) Trends in maternal mortality: 1990 to 2010 - WHO, UNICEF, UNFPA, and The World Bank estimate. Report. The World Health Organization.

17. Liu L, Johnson HL, Cousens S, Perin J, Scott S, et al. (2012) Child Health Epidemiology Reference Group of WHO and UNICEF. Global, regional, and national causes of child mortality: An updated systematic analysis for 2010 with time trends since 2000. Lancet 379: 2151-2161.

18. Pal-de Bruin KMV, Verlinden DA, Jansen YJFM, Theunissen MHC, Broerse A, et al. (2011) Implementation of preconception care in the region Leiden], Report, The Netherlands Organization for Applied Research TNO. 